\section{The National Violent Death Reporting System: a new gold standard for the surveillance of violence related deaths?}

\section{A Butchart}

\section{From its modest beginnings based on the analysis of death certificates, the National Violent Death Reporting System represents what can be achieved through sustained investment in efforts to measure violence related mortality}

T he US National Violent Death Reporting System (NVDRS) is a major innovation in violent death surveillance. The NVDRS links data for individual cases from multiple sources, and, where more than one death is associated with an incident, links records for all deaths that occurred in the incident. The NVDRS thus makes it possible to answer questions that are critical for the development of effective violence prevention strategies. What are the risk factors for multiple homicides? What proportion of people who commit homicide go on to kill themselves? How frequently are homicides associated with child maltreatment? Is the national level of violent death in schools increasing or decreasing? What proportion of homicides is associated with illicit drug deals? By having trained coders extract data from death certificates, medical examiner, coroner, and law enforcement records, the NVDRS provides the information sooner than vital statistics data, which are available only two years after a death. As the articles in this special issue illustrate, the combination of comprehensiveness and timeliness mean that NVDRS data are in high demand by violence prevention practitioners. As such, the NVDRS is the new gold standard for violent death surveillance systems elsewhere. Widespread dissemination about how the NVDRS works and its benefit for violence prevention will hopefully lead other countries that already have the elements of such a system in place to emulate the NVDRS.

In a global perspective, however, only a small proportion of low, middle, and high income countries have in place the elements needed for an NVDRS-type system. The cause of death statistics that countries submit to the World Health Organization (WHO) provide an indicator of violent death reporting coverage. This shows that just a third of the world's population is covered by systems that capture sufficient detail about injury related deaths to permit their analysis by intent (intentional, unintentional, undetermined), underlying cause (for example, firearm, poisoning, sharp force) and manner of death (for example, homicide, suicide, legal operation, war). Regionally, this level of coverage extends through North America and much of Latin America and the Caribbean; Western Europe and much of Eastern Europe; Scandinavia, and a few countries in Asia and the Pacific, such as Australia, Japan, New Zealand, and Thailand. For most of Africa, the Middle East, and Asia, and therefore for some of the regions with the highest estimated violent death rates, cause of death statistics lack even the detail required to discriminate between intentional and unintentional injury deaths. ${ }^{1}$ There is thus a wide gap between the NVDRS that uses death certificates as one among other data absence of even violent death certification systems for the majority of the world's population. This raises questions about how to frame discussions of the NVDRS so that it will inspire countries which are now only beginning to establish violent death certification systems, and for which the prospect of trying to move instantly from nothing to a sophisticated multisource violent death reporting system could prove daunting.

First, it is important to emphasize that the NVDRS evolved over time. The origins of the NVDRS can be traced back to the use of death certificates to examine demographic patterns in risks and trends. Then, as the importance of violent deaths became more established, so the need for a more complete description was recognized and police data were added into the picture. In the sources, and what is effectively an late 1990s further data sources were linked into what was then called the National Violent Injury Statistics System, ${ }^{2}$ and, based upon its success, political and financial support for the NVDRS was obtained in 2002. This implies that while some countries could leapfrog the initial and intermediary stages and go straight to an NVDRSstyle system, even the investigation and certification of violent deaths for only a limited sector of the population can be regarded as laying a foundation for subsequent developments towards a more representative and comprehensive system. In Mozambique, for example, a 10 year retrospective review of data for the Maputo City mortuary helped to convince policy makers of the value in investing in ongoing prospective data collection, and since 2005 a fatal violence and injury surveillance system has been established for Maputo City, ${ }^{3}$ with plans to expand the system to other cities and regions of Mozambique as their medico-legal capacity becomes sufficiently developed.

A second observation is that the NVDRS has developed within a strong violence prevention culture that dates back to the early 1980s and has demanded increasingly detailed information about violence to inform and monitor local, state, and national violence prevention policy making and programming. ${ }^{4}$ This highlights the importance of interest on the part of policy makers in having detailed information about the contexts, causes, and consequences of violent deaths. Elsewhere in the world, as evidenced by examples from Jamaica ${ }^{5}$ and South Africa, ${ }^{6}$ high levels of violence stimulated top level government interest in using existing services as a basis for the establishment of a system dedicated to the ongoing collection of information on injury related deaths (in South Africa) and on non-fatal violent injuries presenting at hospital emergency departments (Jamaica). In both countries, the political support that enabled these developments came when homicide rates were already at high levels, and violent injury surveillance systems that should have been in place decades sooner to provide early warning were developed in reaction to a problem that had already grown to massive proportions. Many other countries that have yet to develop violent death reporting systems are probably also already experiencing high rates of violent deaths, and while their value for preventing future violence means it is never too late to invest in such systems, the ideal is to start before violent death rates reach desperate levels. 
A final observation is that the NVDRS is possible only because of agreements that permit state public health departments to retrieve, collate, and analyse information relevant to violent deaths from different sectors, including police and criminal justice departments. Assembling data collected by different sectors can only be done by a single agency with the requisite technical competencies, such as a public health department with the capacity for epidemiological surveillance. Although this is self-evident, in many low, middle, and high income countries a territorial approach to data inhibits the sharing of existing information and precludes obtaining a comprehensive picture of the risk factors and circumstances that sustain violent deaths. Agencies embarking on the establishment or improvement of violent death reporting systems should therefore pay careful attention early in the process to ensuring data accessibility and data sharing. Guarantees that all items which could identify the deceased or others involved in any incident will be excluded from published findings and datasets are essential, while clearly demonstrating how the joined-up perspective adds value to each contributing department provides a positive incentive.

In a world where two thirds of the population have yet to be monitored by cause of death investigation and certification systems of sufficient sensitivity to permit the identification of violent deaths, the NVDRS may at first glance appear beyond the reach of many countries. However, close attention to development of the NVDRS shows how it has evolved into its current shape from modest beginnings based upon the analysis of death certificates. The more data about violent deaths became available, the greater was the political interest in obtaining and using such data for prevention. Even for countries only now beginning to develop low cost sample registration systems, the NVDRS therefore represents a valuable example of what can be achieved through sustained investment in efforts to measure violence related mortality.

Injury Prevention 2006;12(Suppl II):ii63-ii64. doi: 10.1136/ip.2006.013516
Correspondence to: Dr A Butchart, Prevention of Violence, Department of Injuries and Violence Prevention, World Health Organization, 20 Avenue Appia, 1211 Geneva 27, Switzerland; butcharta@who.int

Accepted 3 August 2006

Funding: none

Competing interests: none

\section{REFERENCES}

1 Mathers C, Ma Fat D, Inoue M, et al. Counting the dead and what they died of: an assessment of the global status of cause of death data. Bull World Health Organ 2005;83:171-7.

2 Azrael D, Barber C, Mercy J. Linking data to save lives: recent progress in establishing a National Violent Death Reporting System. Harvard Health Policy Review 2001 ; 2:38-41.

3 Nizamo H, Meyrowitsch DW, Zacarias E, et al. Mortality due to injuries in Maputo City, Mozambique. Int $J$ Inj Contr Saf Promot 2006;13:1-6

4 Rosenberg MR, Fenley MA, eds. Violence in America: a public health approach. New York: Oxford University Press, 1991.

5 Hasbrouck LM, Durant T, Ward E, et al. Surveillance of interpersonal violence in Kingston, Jamaica: an evaluation. Inj Control Saf Promot 2002;9:249-53.

6 Butchart A, Peden M, Matzopoulos R, et al. The South African National Non-Natural Mortality Surveillance System-rationale, pilot results and evaluation. S Afr Med J 2001;91:408-17. 